\title{
Optimized Traffic Profile for Flexgrid Optical Networks
}

\author{
Fengqing Liu ${ }^{1}$ and Jaume Comellas ${ }^{2}$ \\ ${ }^{1}$ School of Opto-Electronic Engineering, Nanjing University of Posts and Telecommunications, \\ 66 Xin Mofan Road, Nanjing, 210003,Jiangsu Province, P. R. China \\ E-mail: liufq@njupt.edu.cn \\ ${ }^{2}$ Dept. of Signal Theory and Communications, Universitat Politècnica de Catalunya, \\ UPC Campus Nord, c/Jordi Girona 1, 08034, Barcelona, Spain \\ Email: comellas@tsc.upc.edu, \\ Telephone: +34 934016 441, Fax: +34 934017200
}

\begin{abstract}
This work is focused on studying the connections bandwidth distribution effects on the FlexGrid network performance. An accurate study of spectrum occupancy in heavy loaded FlexGrid optical networks is carried out. This analysis is useful to figure out which traffic profiles are more suitable for this kind of networks. Two different cases are considered with respect to the offered connections. In the Elastic case, connections sizes can take any value from 1 to a maximum number of spectrum slots. In the MultiRate case, only three connections sizes are allowed. It is demonstrated that by generating the connections with some specific statistical properties, the network performance is improved. These properties are derived from the theoretical study of spectrum occupation in ideal conditions. The main contribution of this work is therefore the proposal of an Optimum traffic profile which allows to efficiently using the spectrum in FlexGrid optical networks. The gain obtained by generating the proposed traffic profile is evaluated by means of simulations at the link as well as at the whole network level.
\end{abstract}

Keywords: FlexGrid Optical Networks; Routing and Spectrum Assignment; Network Performance Evaluation 


\section{Introduction}

Due to the continuous advent of new emerging services mainly related to highdefinition video distribution, the IP traffic volume has been exponentially increasing to date. New hardware advances such as multicore processing, virtualization and network storage will support a new generation of e-Science and cloud applications requesting data flows of $10 \mathrm{Gbps}$ up to terabit level. Consequently, network providers will require a new generation of optical transport networks in the near future, so as to serve this huge and heterogeneous volume of traffic in a cost-effective and scalable manner [1]. The elastic optical network (EON) architecture has been proposed [2, 3] as the solution to these large capacity and diverse traffic granularity needs of the future Internet.

Traditional WDM optical networks show a rigid spectrum grid where each optical channel is allocated in a $50 \mathrm{GHz}$ slot. The main EON characteristic consists in breaking this grid and allocating the appropriately sized optical spectrum to each connection. Assuming that connections range from $10 \mathrm{Gbps}$ to even $1 \mathrm{~Tb} / \mathrm{s}$, the spectrum is typically divided in $12.5 \mathrm{GHz}$ Frequency Slots (FS), and the suitable number of FS is assigned to each connection. The advantages of the EON are twofold: small connections can be served with less than $50 \mathrm{GHz}$ (for example, one single $12.5 \mathrm{GHz}$ slot is enough for a 10 Gbps connection), and, super-channels are spectrum efficiently served (as can be found in [3], 7 FS are enough to allocate a 400 Gbps connection assuming dual polarization and QPSK modulation format). Nevertheless, EON concept introduces additional challenges to the networking level, especially on the efficient connection establishment. Similar to WDM networks, an elastic optical connection must occupy the same spectrum portion between its end-nodes, that is, ensuring the so called spectrum continuity constraint. In addition, the entire bandwidth of the connections must be contiguously allocated, also referred as the spectrum contiguity constraint. The Routing and Spectrum Allocation (RSA) problem in FlexGrid optical networks has been widely studied, considering the static traffic case (network planning phase) as well as dynamic (in operation) network scenarios [3-13]. There, connection arrival and departure processes are random and the network has to accommodate incoming traffic in real time. In a dynamic scenario, the spectral resources allocated to connections are released upon tear down, becoming available for future requests. The randomness in the connections birth and death processes leads to fragmentation $[6,14]$ of the spectral resources in the network, which is an important drawback of EONs.

An accurate modeling of the link occupancy is of capital importance, as it will help in better understanding ways to reduce fragmentation and, in general, to improve the EON performance. Some previous works have dealt with this issue. A link model based on a Continuous-Time Markov Chain (CTMC) was introduced by Yu et al. in [15], with the aim of mathematically describing the differences between traditional WDM networks and FlexGrid networks. Beyranvand et al. in [16] also tackle the problem using Markov chains, in this case from both the node and the whole network points of view, and using different spectrum assignment strategies. In [17], extending the CTMC model previously commented, some conclusions about the best distribution of connections in a FlexGrid spectrum window were extracted. After an accurate analysis of link occupation, it was found in [17] that the number of connections with different sizes (from 1 to $N$ FS), in a perfectly full spectrum window, follow the Golden ratio. This work further extends the outcomes of [17] and tackles the problem with a different approach, by assuming that guard-band FS are encompassed in the connections. This feature allows to actually considering any connection size from 1 to $N$ FS. As will be seen in next sections, this 
assumption leads to interesting results that will be assessed by means of extensive simulations.

This paper addresses the accurate analysis of spectrum occupation in FlexGrid optical networks, where the spectrum continuity and contiguity constraints are met. Two different scenarios are considered with respect to the connections distribution: elastic case, where connections size can take any value from 1 to N FS, and Multi-Rate case, where connections bandwidth can only take 3 values (corresponding to 100Gbps, 400Gbps and 1Tbps connections). Connection arrival and departure processes are random at any specific traffic intensity, and main objective of this work is to find the optimum distribution of different sized connections which allows minimizing the bandwidth blocking probability in the network.

The rest of paper is organized as follows. Section II is devoted to the analysis of link spectrum occupation. Different subsections are dedicated to the study of the Elastic and the Multi-Rate cases. An analysis of the required traffic characteristics to efficiently use the spectrum is also introduced in this Section. In Section III simulations at the link and network level assess the validity of the concepts previously presented. Finally, Section IV concludes the paper.

\section{Full spectrum state and suitable traffic profiles in FlexGrid networks}

As has been mentioned above, the links available bandwidth in FlexGrid networks is divided into frequency slots (FS), whose typical size is $12.5 \mathrm{GHz}$ (other values such as $6.25 \mathrm{GHz}$ can also be used). If a $4 \mathrm{THz}$ spectrum is considered in each link, there are 320 FS (assuming $12.5 \mathrm{GHz}$ per FS) available to be assigned to the requested connections. The appropriate number of contiguous FS is assigned to fulfill the bandwidth requirements of connections, and some FS are usually reserved between adjacent connections as guard-bands. In this paper, the guard-band bandwidth is assumed as part of the spectrum allocated to each connection, in contrast with the assumptions taken in [17]. This means that if the required bandwidth of a connection is $n$ FS, the guard-band bandwidth is assumed as being part of these $n$ FS.

Each link can be viewed as an $N$ FS spectrum window, where $N$ is the number of FS available in the link (as stated before, $N=320$ FS will be considered during simulations). Depending on the load offered to the network, a given FS at any link can be either available or occupied. This work takes as the starting point the analysis of a perfectly filled spectrum (hereafter called F state), in which all the FS of the link are used by connections. This idea was previously introduced in [17] and demonstrated to be useful to deduce suitable properties of the load offered to FlexGrid optical networks.

Concerning connection demands, two different traffic scenarios are analyzed: the first one is the Elastic traffic scenario, where any connection bandwidth size from 1 to $S$ FS (with $S \leq N$ ) is allowed; the second one is a Multi-Rate scenario where the connections sizes are limited to some selected sizes corresponding, for example, to $100 \mathrm{Gbps}, 400$ Gbps and 1 Tbps connections. It is noteworthy that the relation between spectrum occupied by connections and their bitrate depends actually on the optical path length. Higher number of bits per symbol can be used for shorter transmission distances (so the spectral efficiency becomes higher). As stated previously, the main objective of this work is finding the optimum distribution of connection sizes and the ratio between spectrum consumed and traffic carried is only used in this case as a numerical example 
taken from [3]. Nevertheless, the modulation format vs. reach problem in EONs is out of the scope of this work whose focus is on spectrum usage.

\subsection{Analysis of the Elastic scenario}

In this case, any connection size from 1 FS to $S$ FS will be offered to the network. For a fixed bandwidth window, there are many different connections combinations that lead to the F spectrum state. As an example, Figure 1 shows the possible combinations that lead an $N=4$ FS window to the $\mathrm{F}$ spectrum state. It also gives exact values to the probability of each connection size being present in the F spectrum state. It has to be highlighted that the entire bandwidth of any connection must be contiguously allocated. That is, the spectrum contiguity constraint must be met when allocating spectrum slots for a connection. When allocating spectrum in the network paths, every connections must occupy the same spectrum portion throughout the whole path (spectrum continuity constraint).

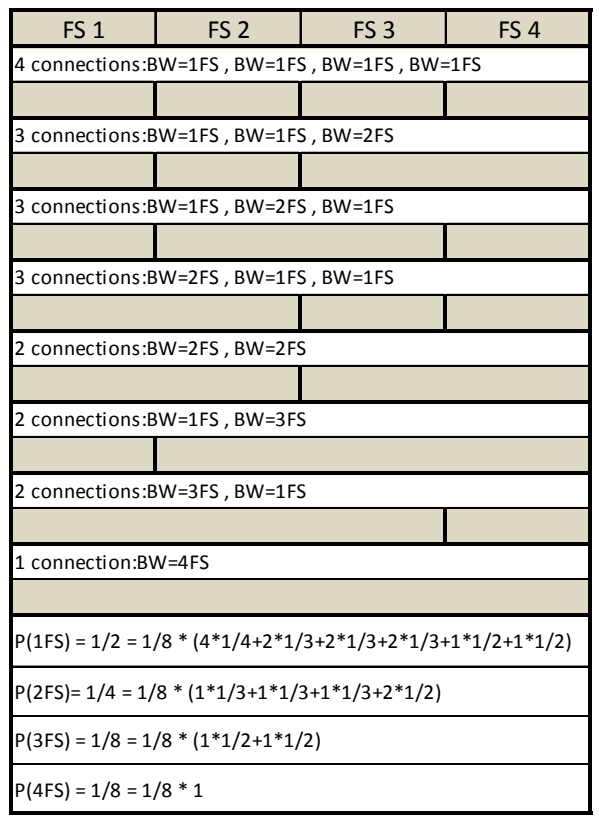

Fig. 1. Connections combinations that lead a 4 FS spectrum window to the F state. Probabilities of presence of the different connections sizes in F state are shown in last four rows.

It is noteworthy that in the Fig. 1 example, $N=S=4 \mathrm{FS}$; nevertheless, the analysis done can be extended to a scenario where $N>S$ without loss of generality. Considering this 4 FS window, it is found that there are 8 different connections combinations (depicted in Fig. 1) that lead it to the F state. The probabilities of finding 1FS, 2FS, 3FS and 4FS connections in this F state are, respectively, 1/2, 1/4, 1/8 and 1/8.

The connections combinations leading to the F state satisfy the equation $\sum_{i=1}^{4} i \cdot x_{i}=4$ where $x_{i}(1 \leq i \leq 4)$ corresponds to the number of connections with size $i$ FS present in that F state. If a tuple $\left(x_{1}, x_{2}, x_{3}, x_{4}\right)$ is used to express each solution, five different solutions are found for the $N=4 \mathrm{FS}$ window, whose values are $(4,0,0,0),(2,1,0,0),(0,2,0,0)$, $(1,0,1,0)$ and $(0,0,0,1)$. There can be several connections combinations leading to some 
of these solutions. For example, when considering solution $(2,1,0,0)$, there are 3 different connections combinations which include two 1 FS and one 2 FS connections, as depicted in Fig.1. For a generic solution $m$, the number of different combinations will be denoted by numCombs $(m)$. Considering again the exemplary 4 FS window, the values of $\operatorname{numCombs}(m)$ for $m=1,2,3,4$ and 5 are, respectively, 1, 3, 1, 2 and 1.

By extending this analysis to a generic $N$ FS spectrum window, and assuming that the maximum connection size $(S)$ is equal to $N$ FS, the number of different connections combinations leading to the $\mathrm{F}$ state is found to be $\sum_{m=1}^{M}$ numCombs $(m)=2^{N-1}$, where $M$ is the number of solutions $\left(x_{1}, x_{2}, \cdots, x_{N}\right)$ that satisfy the equation $\sum_{i=1}^{N} i \cdot x_{i}=N$.

In a generic case where the window has N FS and the maximum connection size is $\mathrm{S}$ FS, a tuple $\left(x_{1}, x_{2}, \ldots, x_{\mathrm{S}}\right)$ can be used to express any solution of $N=\sum_{j=1}^{S}\left(j \cdot x_{j}\right)$, where $x_{\mathrm{j}}\left(0 \leq x_{j} \leq N\right)$ is the number of connections of size $j$ FS present in that solution. If there are $M$ different solutions, the $m$-th solution can be denoted as $\left(x_{1}(m), x_{2}(m), \ldots\right.$, $\left.x_{S}(m)\right),(1 \leq m \leq M)$. The number of different combinations corresponding to this $m$-th solution can be calculated as numCombs $(m)=\prod_{j=1}^{S} C_{\text {sum }_{j}(m)}^{x_{j}(m)}$, where $\operatorname{sum}_{j}(m)=\sum_{i=j}^{S} x_{i}(m)$, and $C_{\text {sum }_{j}(m)}^{x_{j}(m)}=\operatorname{sum}_{j}(m) ! /\left\{\left[\operatorname{sum}_{j}(m)-x_{j}(m)\right] ! \cdot x_{j}(m) !\right\}$. If $\beta_{m, k}$ is the appearance probability of the $k$-th combination of solution $m$, and $\gamma_{m, k, j}$ is the appearance probability of a $j$ size connection in the $k$-th combination leading to solution $m$, the probability that at least one $n$ FS connection is present in this F state is given by,

$$
P_{n}=\sum_{m=1}^{M} \sum_{k=1}^{\text {numCombs }(m)}\left\{\beta_{m, k} \cdot x_{n}(m) \cdot \gamma_{m, k, n} / \sum_{j=1}^{S}\left[x_{j}(m) \cdot \gamma_{m, k, j}\right]\right\} \quad 1 \leq n \leq S
$$

For all of the connections size values $(j)$, there is the same $x_{j}(m)$ number of connections in any of the possible combinations leading to the $m$-th solution. Therefore, it can be assumed that each one of the combinations leading to this $m$-th solution has the same appearance probability $\alpha_{m}$. In addition, we assume that the appearance probability of different size connections in the $k$-th combination of solution $m$ is uniformly distributed, so $\gamma_{m, k, j}=\gamma_{m, k, i}$, where $i$ and $j$ can take any value between 1 and $S$. When considering these two assumptions, the following expression is derived:

$$
P_{n}=\sum_{m=1}^{M}\left(\alpha_{m} \cdot \operatorname{numCombs}(m) \cdot x_{n}(m) / \sum_{j=1}^{S} x_{j}(m)\right) \quad 1 \leq n \leq S
$$

If any of the solution combinations are considered to be equally probable, namely $\alpha_{m}$ is a constant $1 / \sum_{m=1}^{M} n u m \operatorname{Combs}(m)$, Eq.(2) can be expressed as 


$$
P_{n}=\left(1 / \sum_{m=1}^{M} n u m \operatorname{Combs}(m)\right) \cdot \sum_{m=1}^{M}\left[n u m \operatorname{Combs}(m) \cdot x_{n}(m) / \sum_{j=1}^{S} x_{j}(m)\right] \quad 1 \leq n \leq S
$$

For the previously discussed case where $S=N=4$ FS, it is found that $\sum_{m=1}^{M}$ num $\operatorname{Combs}(m)=2^{(N-1)}$ and, assuming that the different combinations probability is uniformly distributed, $\alpha_{m}=2^{-(N-1)}=2^{-3}=1 / 8$. All the different combinations in this exemplary case are enumerated and the occurrence probability of each connection size is given. Results assuming other combinations probability values, which would correspond to different traffic profiles, can be similarly derived by using Eq. (1) and (2).

When all the solutions combinations are equally probable, the probability that the $\mathrm{F}$ state includes at least $1 \mathrm{FS}$ connection is equal to $1 / 2$, while the probability of other connection sizes appearance can be expressed as

$$
P(n)=\left\{\begin{array}{lr}
(1 / 2)^{n} & 1 \leq n \leq N-1 \\
(1 / 2)^{n-1} & n=N
\end{array}\right.
$$

Eq. (4) can be derived from the following calculation process:

(1) Enumerate all the solutions of $\left\{x_{j}, 1 \leq j \leq N\right\}$ that satisfy $N=\sum_{j=1}^{N}\left(j \cdot x_{j}\right)$.

(2) For each solution of $\left\{x_{j} \mid 1 \leq j \leq N\right\}$, calculate $\operatorname{numCombs}(m)=\prod_{i=1}^{N} C_{\text {sum }_{i}(m)}^{x_{i}(m)}$, where $\operatorname{sum}_{i}(m)=\sum_{j=i}^{N} x_{j}(m)$, and $C_{\text {sum }_{i}(m)}^{x_{i}(m)}=\operatorname{sum}_{i}(m) ! /\left[\left(\operatorname{sum}_{i}(m)-x_{i}(m)\right) ! \cdot x_{i}(m) !\right]$.

(3) Calculate the occurrence probability of each connection size $n$ according to Eq.(3).

It is concluded from Eq. (4), that the appearance ratio between $n$ FS and $n+1$ FS connections is 2 for $1 \leq n \leq N-1$, while the probabilities of finding $N-1$ FS and $N$ FS connections in the $\mathrm{F}$ state are equal to $(1 / 2)^{N-1}$. The following assumptions have been taken into account when calculating these probability ratios:

1) All the connections combinations leading to the F state have uniformly distributed probability of appearance, namely $1 / 2^{\mathrm{N}-1}$;

2) All the connections present in a given combination are equally probable regardless of its size.

It has also been found that there is an interesting relationship between Eq. (4) and the number of $n$ FS connections in the F state, which can be expressed as,

$$
\left\{\begin{array}{l}
f(n)=2 \cdot f(n+1)+2^{N-2-n} \quad n \leq N-2 \\
f(N-1)=2 \\
f(N)=1
\end{array}\right.
$$


where $f(n)$ corresponds to the number connections of size $n$ FS that are present in the F state. For example, using again the $4 \mathrm{FS}$ spectrum window, the number of connections of each size is $f(4)=1, f(3)=2, f(2)=2 \cdot f(3)+2^{4-2-2}=5$ and $f(1)=2 \cdot f(2)+2^{4-2-1}=12$.

It can therefore be concluded that,

$$
\left\{\begin{array}{c}
f(N)=1, \\
f(N-1)=2, \\
f(N) / f(N-1)=1 / 2
\end{array}\right.
$$

The number of connections of size $n \leq N-2$ is given by,

$$
\begin{aligned}
f(n) & =2 \times f(n+1)+2^{N-2-n} \\
& =\cdots \cdots \\
& =2^{N-n-1} \times f(N-1)+(N-n-1) \times 2^{N-2-n} \\
& =2^{N-n}+(N-n-1) \times 2^{N-2-n}
\end{aligned}
$$

Therefore, the ratio between $f(n+1)$ and $f(n)$ corresponds to,

$$
\begin{aligned}
& \frac{f(n+1)}{f(n)}=\frac{2^{N-n-1}+(N-n-1-1) \cdot 2^{N-2-n-1}}{2^{N-n}+(N-n-1) \cdot 2^{N-2-n}} \\
& =\frac{1}{2}-\frac{2^{N-n-3}}{2^{N-n}+(N-n-1) \cdot 2^{N-n-2}} \\
& =\frac{1}{2}-\frac{1}{8+2(N-n-1)}
\end{aligned}
$$

Assuming that $N=4$ and $n \leq N-2$,

$$
\frac{f(n+1)}{f(n)} \geq \frac{1}{2}-\frac{1}{10}=0.4
$$

As a general rule, when $n<<N$,

$$
\frac{f(n+1)}{f(n)} \approx \frac{1}{2}
$$

It is finally concluded that the ratio between the number of $n+1$ FS connections and the number of $n$ FS connections appearing in the F state is always close to 1/2. This relation will be later used to describe the Optimum traffic profile for Elastic optical networks.

\subsection{Analysis for 100 Gbps/400Gbps/1Tbps connections sizes (Multi-Rate case)}

The analysis previously done for a fully elastic scenario has been extended to a multirate case where only some connections sizes are allowed. More concretely, the connections data rates are $100 \mathrm{Gbps}, 400 \mathrm{Gbps}$ or 1 Tbps. Assuming that the DP-QPSK modulation format is used (the spectrum efficiency is $4 \mathrm{bit} / \mathrm{s} / \mathrm{Hz}$ ), these connections need respectively, $47.5 \mathrm{GHz}, 85 \mathrm{GHz}$ and $200 \mathrm{GHz}$ including guard-bands [3]. Therefore, taking into account that each FS corresponds to $12.5 \mathrm{GHz}, 4,7$ and 16 FS will 
respectively be required to transport these connections. In order to find the different connections combinations that lead to a perfectly full spectrum (F state), the following equation applies,

$$
4 \cdot x_{1}+7 \cdot x_{2}+16 \cdot x_{3}=N
$$

By solving Eq. (11) with $N=320$, it is found from that there are $M=132$ different solutions. For each specific value of $x_{1}(m), x_{2}(m)$ and $x_{3}(m),(1 \leq m \leq M)$, and defining $\operatorname{sum}(m)=\sum_{i=1}^{3} x_{i}(m)$, the number of combinations satisfying each one of the solutions is,

$$
\operatorname{num} \operatorname{Combs}(m)=C_{\operatorname{sum}(m)}^{x_{1}(m)} \cdot C_{\text {sum }(m)-x_{1}(m)}^{x_{2}(m)} \cdot C_{x_{3}(m)}^{x_{3}(m)}
$$

or

$$
\operatorname{numCombs}(m)=\prod_{i=1}^{3} C_{\operatorname{sum}_{i}(m)}^{x_{(}(m)}
$$

where $\operatorname{sum}_{i}=\sum_{j=i}^{3} x_{j}(m)$ for $1 \leq i \leq 3$.

Using again Eq. (4), the probabilities of appearance in the F state of connections with size 4, 7 and 16 FS (denoted, respectively, as $p_{1}, p_{2}$ and $p_{3}$ ), can be calculated as,

$$
p_{i}=\left[1 / \sum_{m=1}^{M} n u m \operatorname{Combs}(m)\right] \cdot \sum_{m=1}^{M}\left[\operatorname{num} \operatorname{Combs}(m) \cdot x_{i}(m) / \sum_{j=1}^{3} x_{j}(m)\right]
$$

It is found that the probabilities for the three considered connection sizes are, $\mathrm{p}_{1}=55.3 \%, \mathrm{p}_{2}=35.4 \%$ and $\mathrm{p}_{3}=9.3 \%$. These values will be used in next subsection to generate the Optimum traffic profile for the Multi-Rate case, because it can be expected that, by generating traffic with this distribution, the $\mathrm{F}$ state which corresponds to a perfectly occupied spectrum, will be more likely achieved.

\subsection{Optimum traffic profiles in FlexGrid networks}

Based on the above analysis, Optimum traffic profiles have been created trying to fit the appearance probabilities previously discussed. In addition to these Optimum traffic profiles, other profiles have been tested for benchmarking purposes. The different profiles used are detailed in Table 1, where Golden profile was found in [17] as the optimal profile (1 FS connections were not allowed in that case), while TP3 consists in a slight variation of the theoretically found as Optimum in this work and has been included in this study because its statistical parameters are close to those of the Optimum profile. The main difference between Optimum and TP3 profiles is found in the proportions of the different sizes connections (see Table 1). The purpose of TP3 is to consider a profile whose average number of FS per connection is very close to that of the Optimum profile. This way the advantage of the lower blocking experienced by small size connections of the Optimum profile is eliminated. The Uniform profile is typically used in EON simulations and has also been included here for the sake of comparison. A similar rationale has been followed for the Multi-Rate case where 100G, 400G and 1Tbps connections are simulated. 
Average bandwidth sizes for each traffic profile are also shown in Table 1. They have been found as the sum of the ratio of connections of each size multiplied by its bandwidth. For example, the Uniform average size is found as $0.1(1+2+\ldots+10)=5.5$. It is worth noting that this Uniform profile shows always a notably higher average bandwidth value. This is due to the fact that a higher number of large connections are generated in this case, and, taking into account the spectrum contiguity requirement of FlexGrid spectrum assignment, larger connections usually experience higher blocking probability than smaller ones [18].This is a clear drawback of the Uniform profile.

TABLE $1 . \quad$ TRAFFIC PROFILES FOR ELASTIC AND MULTI-RATE CASES

\begin{tabular}{|c|c|c|c|c|c|c|c|c|c|c|c|}
\hline \multicolumn{11}{|c|}{$\%$ of connections } & Average Size (FS) \\
\hline \multicolumn{12}{|c|}{ ELASTIC } \\
\hline Size (FS) & 1 & 2 & 3 & 4 & 5 & 6 & 7 & 8 & 9 & 10 & \\
\hline Uniform & 10 & 10 & 10 & 10 & 10 & 10 & 10 & 10 & 10 & 10 & 5.5 \\
\hline Optimum & 50 & 25 & 12 & 6 & 3 & 2 & 1 & 0.5 & 0.25 & 0.25 & 2.03 \\
\hline Golden & 38 & 24 & 15 & 9 & 6 & 4 & 2 & 1.5 & 0.8 & 0.5 & 2.5 \\
\hline TP3 & 50 & 38 & 1.5 & 1.5 & 1.5 & 1.5 & 1.5 & 1.5 & 1.5 & 1.5 & 2.04 \\
\hline \multicolumn{12}{|c|}{ MULTI-RATE } \\
\hline Size (Gb/s) & \multicolumn{3}{|c|}{100} & \multicolumn{3}{|c|}{400} & \multicolumn{4}{|c|}{1000} & \\
\hline Uniform & \multicolumn{3}{|c|}{33.3} & \multicolumn{3}{|c|}{33.3} & \multicolumn{4}{|c|}{33.3} & 9 \\
\hline Optimum & \multicolumn{3}{|c|}{55.3} & \multicolumn{3}{|c|}{35.4} & \multicolumn{4}{|c|}{9.3} & 6.18 \\
\hline Golden & \multicolumn{3}{|c|}{49.6} & \multicolumn{3}{|c|}{31} & \multicolumn{4}{|c|}{19.4} & 7.26 \\
\hline TP3 & \multicolumn{3}{|c|}{70} & \multicolumn{3}{|c|}{15} & \multicolumn{4}{|c|}{15} & 6.25 \\
\hline
\end{tabular}

Traffic profiles for the Multi-Rate case are given in Table 2, including connections distribution ratio as well as actual traffic carried by each of the connections types.

TABLE 2. TRAFFIC PROFILE FOR MULTI-RATE CASE (INCLUDING CONNECTION RATIOS AND TRAFFIC RATIOS)

\begin{tabular}{|c|c|c|c|c|}
\hline \multicolumn{2}{|c|}{ Size(Gb/s) } & 100 & 400 & 1000 \\
\hline \multirow{3}{*}{ Uniform } & $\%$ Conns & 33.33 & 33.33 & 33.33 \\
\cline { 2 - 5 } & $\%$ Traffic & 6.66 & 26.66 & 66.66 \\
\hline \multirow{3}{*}{ Optimum } & \% Conns & 55.3 & 35.4 & 9.3 \\
\cline { 2 - 5 } & \% Traffic & 19.1 & 48.8 & 32.1 \\
\hline \multirow{3}{*}{ Golden } & \% Conns & 49.6 & 31 & 19.4 \\
\cline { 2 - 5 } & \% Traffic & 13.5 & 33.7 & 52.8 \\
& & & & 15 \\
\hline \multirow{2}{*}{ TP3 } & \% Conns & 70 & 15 & 53.6 \\
\cline { 2 - 5 } & \% Traffic & 25 & 21.4 & \\
\hline & & & & \\
\hline
\end{tabular}

Generated traffic is represented in both, percentage of connections as well as actual traffic ratio carried by each of the connection sizes. The latter gives a more intuitive idea about how much traffic is carried by each type of connections. Indeed, it can be seen 
how for Uniform traffic the actual traffic carried by the 100 Gbps connections is just $6.66 \%$ while in terms of connections $1 / 3$ of them have this size. On the other hand, when considering the Optimum profile, more than half of the connections are 100 Gbps while only $19.1 \%$ of the traffic is carried by them.

\subsection{Blocking probability after connection torn-down}

Let us now assume a link in its F state where a 1FS connection ends; the spectrum void left is useless for any arriving connection larger than 1 FS. Extending this obvious statement, voids from 1 to $n-1$ FS are unusable when an $n$ FS connection has to be allocated. In other words, when a 2 FS connection has to be allocated, the probability of finding a useless void is the same as that of 1 FS connection appearance in the F state, which is equal to $1 / 2$ as derived from Eq. (5). For 3 FS connections, the blocking probability is the sum of $1 \mathrm{FS}$ and 2 FS connections appearance $\left(1 / 2+(1 / 2)^{2}=3 / 4\right)$. And for a generic $n$ FS connection, the probability of finding a unusable void is $1 / 2+(1 / 2)^{2}+\cdots+(1 / 2)^{n-1}=1-(1 / 2)^{n-1}$. In other words, the probability of successfully allocating an $n$ FS connection is $1-\left[1-(1 / 2)^{n-1}\right]=(1 / 2)^{n-1}$.

When the offered load value is high, the steady-state of links spectrum is close to this F state. Whether a new $n$ FS connection could be served or not is given by the probability of finding an enough large void, namely $(1 / 2)^{n-1}$.

Table 3 summarizes the previous discussion and gives exact values for the probability of presence of each connection size in the F state, as well as their blocking probabilities after the torn-down of any connection from the F state. It needs to be noted that, the probability of an N FS connection to be present in the F state is $1 / 2^{N-1}$, instead of $1 / 2^{N}$. For example, when $N=4$, the probability of 4 FS connections is $1 / 8=1 / 2^{4-1}$, as denoted by Fig.1. It is obviously found that the blocking probability increases with the connections size. It has been previously demonstrated in FlexGrid networks [18] that the bigger the size of a connection, the bigger its blocking probability (BP) is. This work considers a very specific case where the link is supposed to come from a perfectly occupied spectrum (F state). Although this assumption is unrealistic in actual networks, it is useful to theoretically demonstrate some performance properties of FlexGrid optical networks.

TABLE 3. PROBABILITY OF APPEARANCE AND BLOCKING FOR THE DIFFERENT CONNECTIONS SIZES

\begin{tabular}{|c|c|c|c|c|c|c|c|c|}
\hline Connection size (FS) & 1 & 2 & 3 & $\cdots$ & $n$ & $\cdots$ & $N-1$ & $N$ \\
\hline $\begin{array}{c}\text { Prob. to be present } \\
\text { in F state }\end{array}$ & $1 / 2$ & $1 / 4$ & $1 / 8$ & $\cdots$ & $1 / 2^{\mathrm{n}}$ & $\ldots$ & $1 / 2^{\mathrm{N}-1}$ & $1 / 2^{\mathrm{N}-1}$ \\
\hline $\begin{array}{c}\text { BP (after any } \\
\text { connection ends) }\end{array}$ & 0 & $1 / 2$ & $3 / 4$ & $\cdots$ & $1-\left(1 / 2^{\mathrm{n}-1}\right)$ & $\cdots$ & $1-\left(1 / 2^{\mathrm{N}-2}\right)$ & $1-\left(1 / 2^{\mathrm{N}-1}\right)$ \\
\hline
\end{tabular}


The results obtained in previous paragraphs will be assessed in next Section by means of simulation under realistic load conditions. The main objective is to demonstrate that even the network load is clearly far from the values leading the links to the F state, using the Optimum traffic profile previously envisaged will provide some benefits in terms of network blocking probability at any load.

\section{Simulation Framework and Results}

A Matlab based simulator has been developed to assess the benefits obtained when Optimum traffic profiles are generated. Simulations are carried out at both, a single link as well as the whole network level. Simulator main characteristics are,

- Connections bandwidth can take any value from 1 to $S$ FS. As it has been highlighted in the Introduction, no explicit guard-band is considered between adjacent connections in a link. Current technologies allow for narrow filtering [19], so a complete guard-band FS can be avoided for low rate (e.g. 10 Gbps) connections as they can be efficiently filtered on few $\mathrm{GHz}$ with low penalties.

- Maximum number of FS (MaxFS) in a link is set to 320. It is assumed that the available bandwidth per link is $4 \mathrm{THz}$, and each FS is $12.5 \mathrm{GHz}$. This is a value typically considered as the available spectrum in each fiber, and corresponds to the Cband spectrum [20].

- Connections Inter-Arrival Time (IAT) follows a Poisson process with average value equal to 1; Connections Holding Time (HT) follows a negative exponential distribution, and its average value is adjusted to get the suitable traffic intensity, which is expressed as the offered load.

- The spectrum assignment strategy used is First-Fit, which allocates spectrum slots to the connections as on the left of the link spectrum as possible. When the whole network is considered, K-shortest paths routing (with $\mathrm{K}=3$ ) is used for routing. Each connection will try to be set up in the shortest path first; if not enough large spectrum void is found in the path, next shortest path (up to three times) is chosen.

When a connection arrives, it will be served when there are enough spectrum voids in one of K-shortest paths, otherwise it will be rejected. It is a queuing system with loss of connections, or blocking some connections. Its service intensity is usually represented by HT/IAT. In elastic optical networks, as each connection has a particular bandwidth requirement, and it goes through a given number of links, the service intensity (hereafter called OfferedLoad) of a link should take into consideration the connections sizes, as well as the number of hops that every connection is going through. For statistical purposes, they are represented by their average values. According to the above discussion, offered load to the links is calculated using

$$
\text { OfferedLoad }=\frac{\overline{H T} \cdot \overline{B W} \cdot \overline{\text { hops }}}{\overline{I A T} \cdot \text { MaxFS } \cdot \text { LinkNo }}
$$

where $\overline{H T}$ is the average connections Holding Time, $\overline{B W}$ is the average connections bandwidth, $\overline{\text { hops }}$ is the average number of hops per connection, the average inter-arrival 
time $\overline{I A T}$ is set to 1 , MaxFS is the number of FS per link (so the offered load is normalized to this value), and LinkNo is the number of links in the network.

The throughput is therefore calculated as,

$$
\text { Throughput }=\text { OfferedLoad } \cdot(1-\mathrm{BP})
$$

where BP is the blocking probability.

Two different scenarios have been considered during simulations. First results focus on a single link and their main purpose is to assess the validity of the theoretical results obtained in previous Sections. When this single link case is considered, the values of hops and LinkNo are set to 1 . Later simulations are devoted to the whole network performance using the well-known NSFnet (14 nodes and 21 bi-directional links) and US_Backbone (24 nodes and 43 bi-directional links) topologies [21]. When considering the whole network, the spectrum continuity constraint, whose effects are considerable in the RSA problem, is also taken into account. As for the time complexity analysis, two contributions, stemming from, respectively, routing and spectrum allocation are taken into account. As the shortest path is calculated adaptively (up to three trials in case there is blocking), and as explained in reference [9], its time complexity is $\mathrm{O}\left(\mathrm{k} \cdot \mathrm{L} \cdot \mathrm{N}^{2} \cdot \mathrm{Z}\right)$, where $\mathrm{k}=3, \mathrm{~L}=$ longest route, $\mathrm{N}=$ number of nodes and $\mathrm{Z}=$ number of generated connections. Time consumption from the First-Fit spectrum allocation process is $\mathrm{O}(\mathrm{L} \cdot \mathrm{W} \cdot \mathrm{Z})$ where $\mathrm{W}$ is the number of FS per link.

Simulation results for Elastic traffic (connections with size from 1 to $10 \mathrm{FS}$ are generated) in a single link are summarized in Figures 2 and 3. It is worth noting that each point on all of the results Figures has been obtained by averaging 10 simulations. 95\% confidence intervals are shown on the plots but, for the sake of clarity, these interval representations are avoided when their size is smaller than the markers.

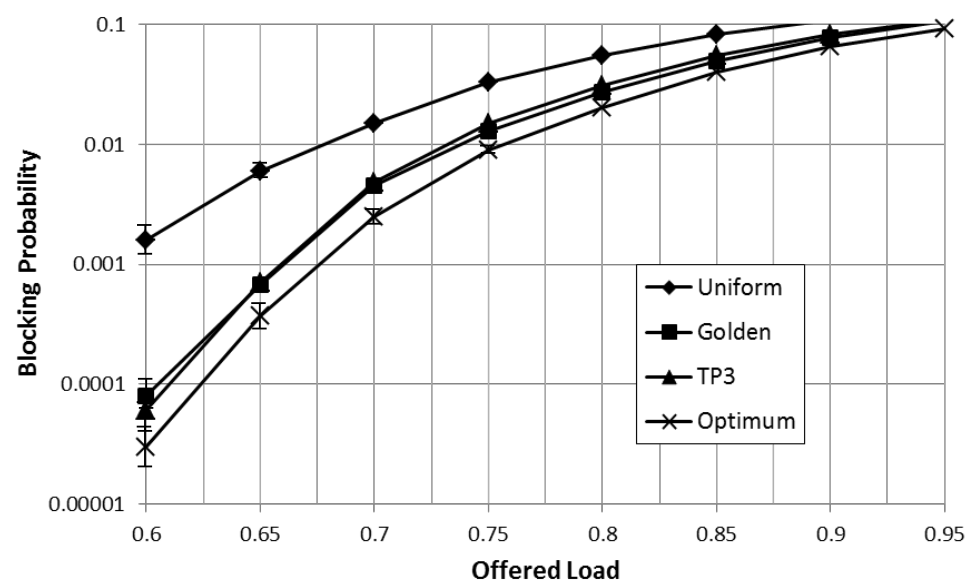

Fig. 2. Link Blocking probability (BP) versus Offered Load for the different traffic profiles considered. Elastic (1 to 10 FS) connections are generated. 
It is clear that the Optimum profile, the one fitting the traffic distribution in the F state, leads to a lower $\mathrm{BP}$ value at any load value. As an example, for $\mathrm{BP}=0.01$, the load for Optimum profile is 0.76 while it is 0.735 for Golden and TP3, and 0.675 for the Uniform profile. It is concluded that when Optimum traffic profile is generated the best link performance is attained. Uniform is the worst case, and Golden performs almost exactly like TP3. It can be concluded from Figure 3 that the Optimum traffic profile allows the highest link throughput.

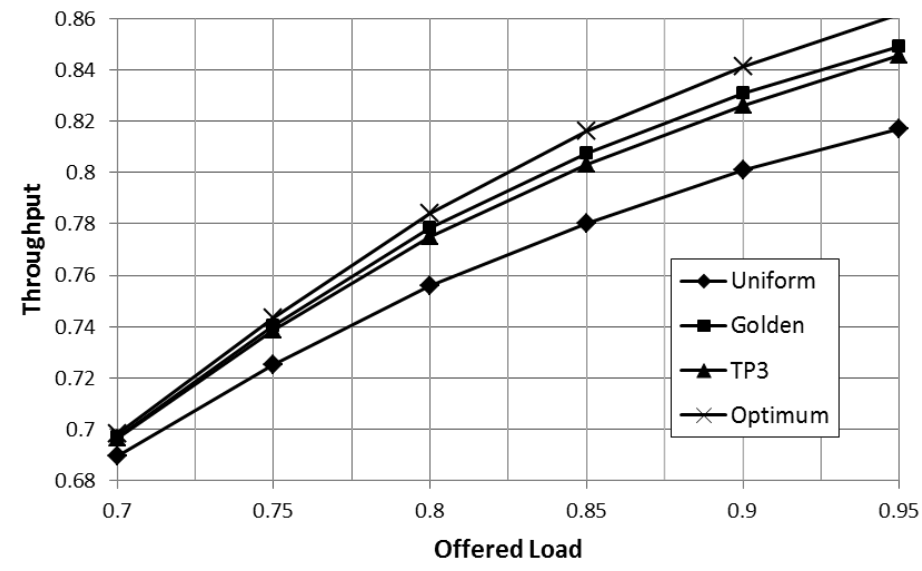

Fig.3. Link Throughput vs. Offered Load for the different traffic profiles considered. Elastic (1 to $10 \mathrm{FS}$ ) connections are generated.

Taking into account the definition of the Offered Load given by Eq. (15), the gain of Optimum with respect to Uniform profile in terms of spectrum used at $\mathrm{BP}=0.01$ is (0.76$0.675) \cdot 320=27.2 \mathrm{FS}$, which, assuming $12.5 \mathrm{GHz}$ per FS, correspond to $340 \mathrm{GHz}$ of additional used spectrum in this single link case. If DP-QPSK is assumed, so the spectral efficiency is close to $4 \mathrm{~b} /(\mathrm{s} \cdot \mathrm{Hz})$, the link would be able to convey an added traffic higher than 1.3 Tbps. When compared with Golden or TP3 (which, as shown in Table 1, have statistical parameters closer to the Optimum profile), this gain is reduced but it is yet notable. Taking again the load value at $\mathrm{BP}=0.01$, it corresponds to about 0.735 in these cases, so the extra link load carried when the Optimum profile is generated, using the same modulation format, is around $400 \mathrm{Gbps}$.

Figure 4 shows the NSFnet network performance. It is noteworthy that the trends previously seen at the link level hold at the whole network scenario when the spectrum continuity is added to the spectrum contiguity constraint. Gain provided by Optimum with respect to Uniform traffic profile has even increased, attaining values around $14 \%$ in terms of network throughput at $\mathrm{BP}=0.01$. 


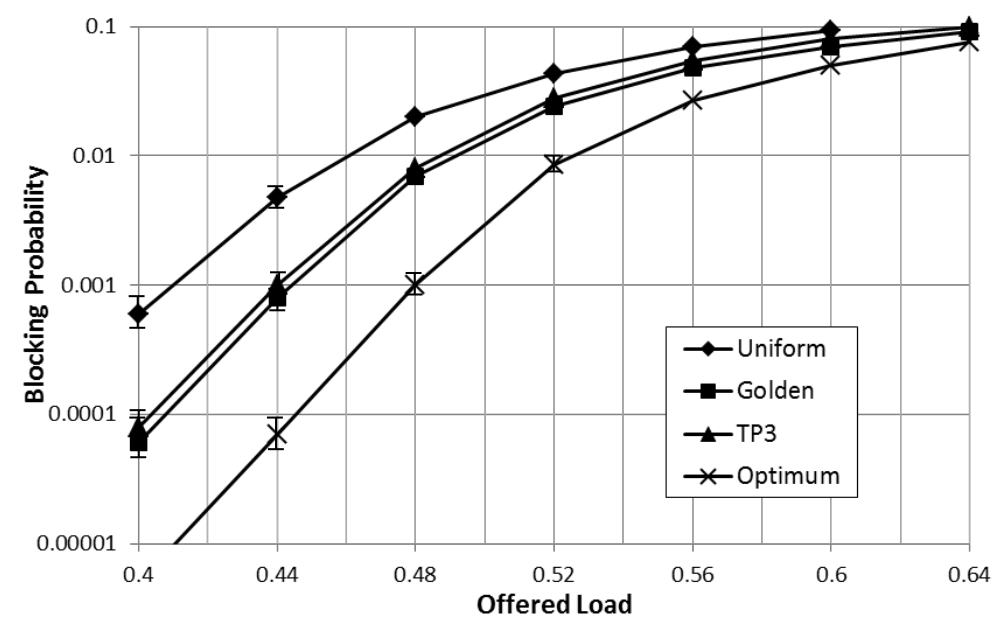

Fig. 4. NSFnet network Blocking probability (BP) versus Offered Load for the different traffic profiles considered. Elastic (1 to 10 FS) connections are generated.

The same trend is observed when the US_backbone topology is simulated, as shown in Figure 5. Nevertheless, some differences are observed in this case:

- The Offered Load values for the blocking probabilities of interest are notably smaller (e.g. load values for $\mathrm{BP}=1 \%$ are around 0.4 while it where close to 0.5 for the NSFNet topology). This is due to the effect of the spectrum continuity constraint, which is harder to satisfy in this larger topology.

- Although Optimum profile shows still clearly superior performance than the rest of profiles, its gain with respect to Uniform profile has been reduced from the $14 \%$ previously commented to around $9 \%$. In any case, it still represents a notable benefit.

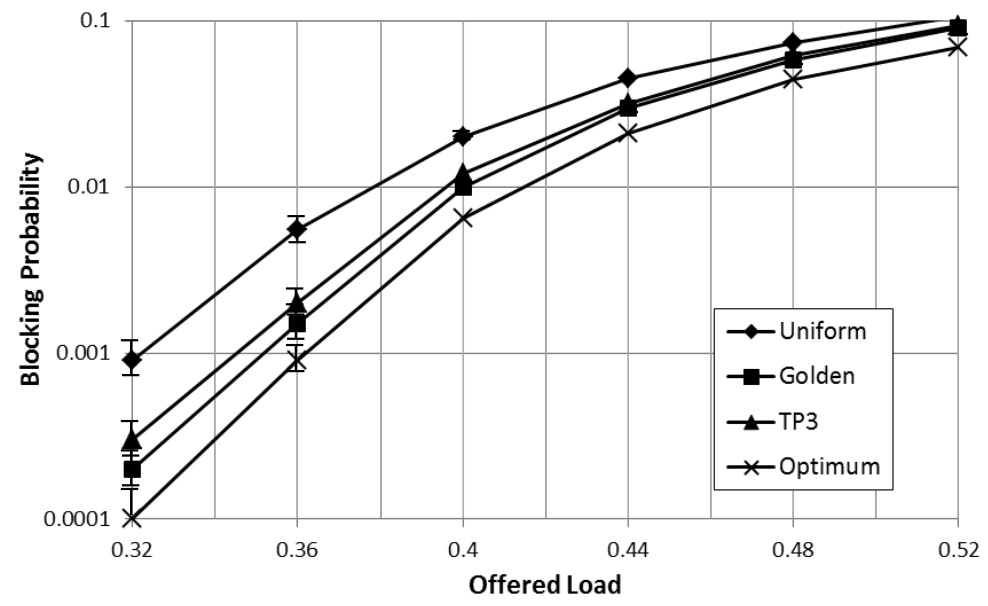

Fig. 5. US_Backbone network Blocking probability versus Offered Load for the different traffic profiles considered. Elastic (1 to 10 FS) connections are generated.

Simulations done for the Multi-Rate case are shown in Figures 6 and 7. As explained before, connection sizes have been limited in this case to 4, 7 and 16 FS (which correspond, respectively, to 100 Gbps, 400 Gbps and 1 Tbps bit-rates). Again Optimum 
traffic profile performs better than Uniform, Golden and TP3 profiles, not only in the link model, but also when the NSFnet and US_backbone network topologies are considered.

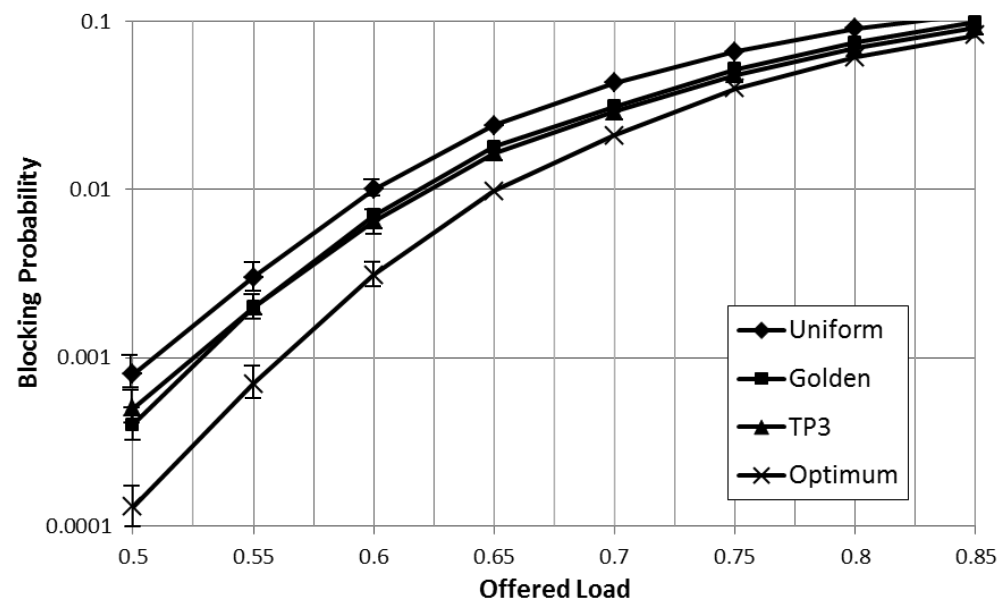

Fig. 6. Link Blocking probability (BP) versus Offered Load for the different traffic profiles considered. Multi-Rate (4 FS, 7 FS and 16 FS) connections are generated.

It has to be highlighted that the supported offered load values for the Multi-Rate case are lower than those for the Elastic one. This is due to the fact that using super-channels (16 contiguous FS are allocated to some connections) implies that the blocking probability, especially for these high bit-rate channels is higher [18]. On the other hand, when using Elastic traffic, an efficient use of the spectrum is guaranteed as any size (from 1 to $10 \mathrm{FS}$ in our case) is generated, so there are much more options than if only three sizes are present. If the supported load for $\mathrm{BP}=0.01$ is considered again in the link scenario (Figure 6), it can be observed how it goes from 0.6 for the Uniform case to 0.65 for the Optimum one. If these values are compared with those obtained in the Elastic case, it is concluded that the gain obtained in relative terms has decreased from $13 \%$ $(0.76 / 0.675)$ to $8 \%(0.65 / 0.6)$.

Finally, Figure 7 shows the results obtained for the Multi-Rate case at the whole network level and considering both topologies. Load values leading to a given BP are even lower in this case because of the network effect (the required FS have to be available on every links along the path). Nevertheless, the main conclusions obtained from previous results hold. Optimum traffic profile has been undoubtedly demonstrated to outperform the rest of the traffic profiles. It is observed again how the gain obtained in the US_Backbone topology is clearly smaller than the one obtained for the NSFNet case. Taking again the load supported values at $\mathrm{BP}=0.01$, this gain is $8.1 \%(0.44 / 0.407)$ for NSFNet, while it is reduced to $5.6 \%(0.356 / 0.337)$ for US_Backbone network. Nevertheless and taking into account the network characteristics (24 nodes, 86 links, 320 FS per link and an average number of hops per connection of 2.99), the gain obtained when the Optimum profile is generated corresponds to $2.2 \mathrm{THz}$ of additional spectrum allocated to the network at $\mathrm{BP}=0.01$. 


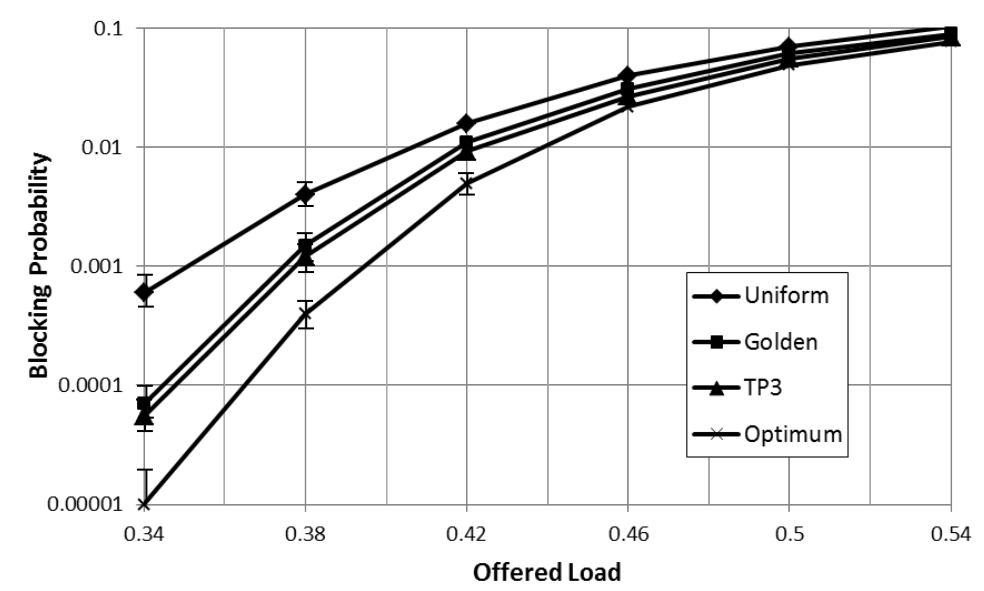

(a)

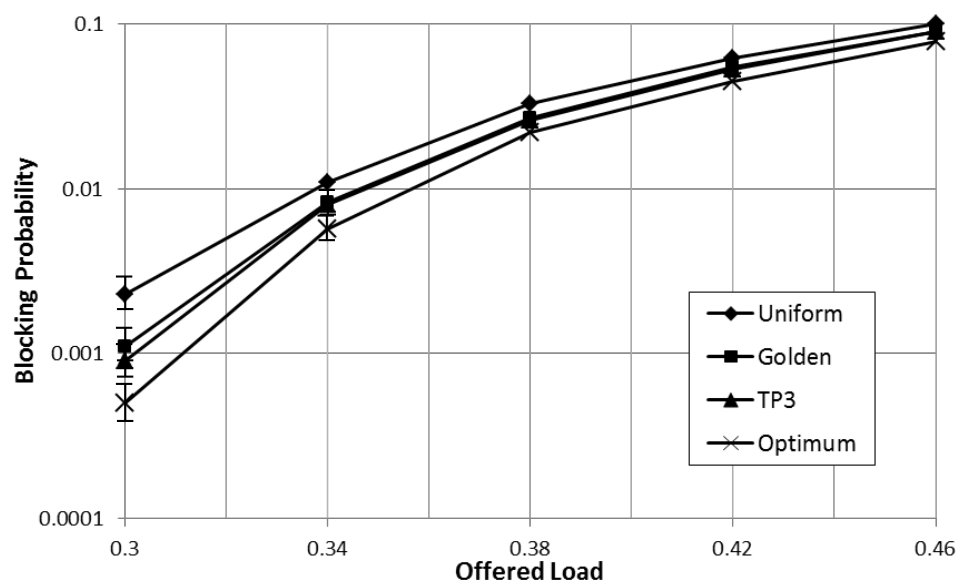

(b)

Fig. 7. Bandwidth Blocking Probability versus Offered Load for the different traffic profiles considered. Multi-Rate connections are generated. (a) NSFNet topology. (b) US_Backbone topology.

The main conclusion after this work is that operation of real FlexGrid optical networks could be optimized by using connections' statistical distributions close to the Optimum profile here disclosed. This means that the network operator should try to distribute the network load in a way as close as possible to this profile. Of course, this will only be possible under certain scenarios, and most of the times other limitations will impose different distribution of the offered connections. On the other hand, as this kind of networks are not yet in the deployment phase, the results here reported can be useful in determining which traffic profiles would allow a better utilization of the available resources. Although traffic profile is always determined by the actual traffic demands, it would be useful to adapt it to the Optimum distribution. This could be achieved by adjusting the number of transceivers of each type in the network nodes to the ratios proposed in this work.

\section{Conclusions}

This work addresses the optimization of traffic profiles in FlexGrid Optical Networks. Two different scenarios have been analyzed: Elastic, where connections can take any size value from 1 to $S$ FS, and Multi-Rate where only three size values are allowed (4, 7 
or 16 FS). Based on the theoretical analysis, optimum connections distributions are proposed. Simulations done at the link as well as at the network level (NSFnet and US_Backbone topologies) assess that the Optimum traffic profile provides better performance in terms of bandwidth blocking probability at any load value. The additional load supported at $\mathrm{BP}=0.01$, compared to other traffic distributions tested, ranges from $5 \%$ to $15 \%$.

\section{Acknowledgments}

This work was supported by the open fund of State Key Laboratory of Advanced Optical Communication Systems and Networks, Shanghai Jiao Tong University, China (No. 2015GZKF03006), and Research Center of Optical Communications Engineering \& Technology, Jiangsu Province (No. ZSF0201). This work has been partially supported by the Spanish MICINN through SINERGY project (TEC2014-59995-R).

\section{References}

[1] Cisco White Paper, "Cisco Visual Networking Index: Forecast and Methodology, 2015-2020", 2016.

[2] M. Jinno, H. Takara, B. Kozicki, Y. Tsukishima, Y. Sone, and S. Matsuoka, "Spectrum-Efficient and Scalable Elastic Optical Path Network: Architecture, Benefits, and Enabling Technologies," IEEE Commun. Mag., vol. 47, no. 11, pp. 66-73, 2009.

[3] O. Gerstel, M. Jinno, A. Lord, B. Yoo, "Elastic Optical Networking: A New Dawn for the Optical Layer," IEEE Commun. Mag., vol. 50, no. 2, pp. s12-s20, 2012.

[4] K. Christodoulopoulos, I. Tomkos, E. Varvarigos, "Dynamic Bandwidth Allocation in Flexible OFDM-based Optical Networks," in Proc. OFC, 2011, OTuI5.

[5] T. Takagi, H. Hasegawa, K. Sato, Y. Sone, B. Kozicki, A. Hirano, M. Jinno, "Dynamic Routing and Frequency Slot Assignment for Elastic Path Networks that Adopt Distance Adaptive Modulation," in Proc. OFC, 2011, OTuI7.

[6] S. Ba, B. C. Chatterjee, S. Okamoto, N. Yamanaka, A. Fumagalli, E. Oki, "Route partitioning Scheme for Elastic Optical Networks with Hitless Defragmentation, " J. Opt. Commun. Netw. vol. 8, no. 6, pp. 356-370, 2016.

[7] B. C. Chatterjee, E. Oki, "Dispersion-Adaptive First-Last Fit Spectrum Allocation Scheme for Elastic Optical Networks," IEEE Commun. Lett., vol. 20, no. 4, pp. 696699, 2016.

[8] W. Fadini, B. C. Chatterjee, E. Oki, "A Subcarrier-Slot Partition Scheme with FirstLast Fit Spectrum Allocation for Elastic Optical Networks," Computer Networks, vol. 91, pp. 700-711, 2015.

[9] B. C. Chatterjee, N. Sarma and E. Oki, "Routing and Spectrum Allocation in Elastic Optical Networks: A Tutorial, " IEEE Communication Surveys \& Tutorials, vol. 17, no. 3, pp. 1776-1800, 2015.

[10] A. Castro, L. Velasco, M. Ruiz, M. Klinkowski, J. Fernández-Palacios, D. Careglio, "Dynamic Routing and Spectrum (Re) Allocation in Future FlexGrid Optical Networks," Computer Networks, vol. 56, no. 12, pp. 2869-2883, 2012. 
[11] L. Gong, X. Zhou, X. Liu, W. Zhao, W. Lu, Z. Zhu, "Efficient Resource Allocation for All-Optical Multicasting Over Spectrum-Sliced Elastic Optical Networks", J. Opt. Commun. Netw. vol.5, no. 8, pp. 836-847, 2013.

[12] J. Zhao, H. Wymeersch, E. Agrell, "Nonlinear Impairment-Aware Static Resource Allocation in Elastic Optical Networks," Journal of Lightwave Technology, vol. 33, no. 22, pp. 4554-4564, 2015.

[13] K. Christodoulopoulos, P. Soumplis, E. Varvarigos, "Planning flexible optical networks under physical layer constraints," J. Opt. Commun. Netw., vol. 5, no. 11, pp. 1296-1312, 2013.

[14] P. S. Khodashenas, J. Comellas, S. Spadaro, J. Perelló, G. Junyent, "Using Spectrum Fragmentation to Better Allocate Time-Varying Connections in Elastic Optical Networks”, J. Opt. Commun. Netw., vol. 6, no. 5, pp. 433-440, 2014.

[15] Y. Yu, J. Zhang, Y. Zhao, X. Cao, X. Lin, W. Gu, “The First Single-Link Exact Model for Performance Analysis of Flexible Grid WDM Networks", in Proc.OFC/NFOEC 2013, JW2A.68.

[16] H. Beyranvand, M. Maier, J. A. Salehi, “An Analytical Framework for the Performance Evaluation of Node- and Network-Wise Operation Scenarios in Elastic Optical Networks”, IEEE Transac. on Comms, vol. 62, no. 5, pp. 1621-1633, 2014.

[17] J. Comellas, M. Ruiz, G. junyent, “Optimizing Link Spectrum Occupancy in Elastic Optical Networks", in Proc. IEEE 16th International Conference on High Performance Switching and Routing (HPSR), Budapest, 2015.

[18] R. Wang, B. Mukherjee, "Spectrum management in heterogeneous bandwidth optical networks", Optical Switching and Networking, vol. 11, pp. 83-91, 2014.

[19] P. S. Khodashenas, J. M. Rivas-Moscoso, D. Klonidis, I. Tomkos, M. B. Shariati, J. Comellas, "Impact of Filter Sharpness on the Performance of Elastic Optical Networks”, in Proc. IEEE International Conference on Communications (ICC), London, 2015.

[20] M. Jinno, B. Kozicki, H. Takara, A. Watanabe, Y. Sone, T. Tanaka, A. Hirano, "Distance-Adaptive Spectrum Resource Allocation in Spectrum-Sliced Elastic Optical Path Network”, IEEE Commun. Mag., vol. 48, no. 8, pp. 138-145, 2009.

[21] Z. Zhu, W. Lu, L. Zhang, N. Ansari, "Dynamic Service Provisioning in Elastic Optical Networks With Hybrid Single-/Multi-Path Routing”, Journal of Lightwave Technology, vol. 31, no. 1, pp. 15-22, 2013. 